\title{
Promising Response to Lenalidomide-Combination
} Therapy in a Discordant Lymphoma Consisting of EBV-Positive Diffuse Large B-Cell Lymphoma and

\section{Angioimmunoblastic T-Cell Lymphoma: A Case Report}

This article was published in the following Dove Press journal:

OncoTargets and Therapy

\author{
Pan $\mathrm{Hu}^{\mathrm{I}, *}$ \\ Yu Ben (D) ${ }^{1, *}$ \\ Juan Liu' \\ Weicheng Zheng (D) \\ Xiyue Yan' \\ Yaping Zhang $\mathbb{D D}^{2}$ \\ Wenyu Shi $\mathbb{I}^{1,2}$

\begin{abstract}
'Department of Oncology, Affiliated Jiangsu, 22600I, People's Republic of China; ${ }^{2}$ Department of Hematology, Affiliated Hospital of Nantong University, Nantong, Jiangsu, 22600I, People's Republic of China

*These authors contributed equally to this work
\end{abstract} \\ Hospital of Nantong University, Nantong,
}

\begin{abstract}
Epstein-Barr virus (EBV)-positive diffuse large B-cell lymphoma (DLBCL) complicated with angioimmunoblastic T-cell lymphoma (AITL) is extremely rare and typically shows an aggressive clinical course and unsatisfactory prognosis. Here, we describe the case of a 77-year-old man who was referred to the hospital because of repeated fever, night sweats, and weight loss. He was finally diagnosed with a discordant lymphoma consisting of AITL and DLBCL, with significantly different maximum standardized uptake values on positron emission tomography/computed tomography. Based on his complex illness and poor performance status, the patient received six cycles of lenalidomide combined with R-miniCHOP regimen and achieved complete remission with tolerable and controlled toxicity. He subsequently received lenalidomide maintenance therapy and achieved sustained remission. We consider the possible causes of this discordance involved AITL and EBVpositive DLBCL, and the possible mechanism of lenalidomide action in both T-cell and B-cell non-Hodgkin lymphomas. Lenalidomide-combination therapy may be a preferable choice in patients with an EBV-associated discordant lymphoma.
\end{abstract}

Keywords: Epstein-Barr virus, diffuse large B-cell lymphoma, angioimmunoblastic T-cell lymphoma, discordant lymphoma, lenalidomide, positron emission tomography/computed tomography

\section{Introduction}

Angioimmunoblastic T-cell lymphoma (AITL) is an aggressive peripheral T-cell lymphoma accounting for $1-2 \%$ of all non-Hodgkin lymphomas. The main clinical manifestations are lymphadenopathy, hepatosplenomegaly, and group B symptoms, while $50 \%$ of the cases also experience skin manifestations and often present with autoimmune diseases. ${ }^{1,2}$ Because of its complex histology and nonspecific clinical features, AITL is often misdiagnosed as other types of lymphoma, and its immunodeficiency may thus subsequently allow infection or reactivation of Epstein-Barr virus (EBV). EBV-infected B cells are indeed present in most AITL cases. ${ }^{3}$ EBV-positive DLBCL is a type of lymphoma with a high incidence in older patients and a dismal outcome. EBV-positive DLBCL accounts for 2-15\% of all DLBCLs worldwide, and these patients generally respond poorly to conventional R-CHOP (rituximab,

Yaping Zhang

Department of Hematology, Affiliated Hospital of Nantong University, Nantong, Jiangsu, 22600I, People's Republic of China

Tel $+86-5|3-8| 160502$

Fax +86-5I3-855I9820

Email zzyaping@163.com 
cyclophosphamide, doxorubicin, vincristine, and prednisone). ${ }^{4} \mathrm{~A}$ small number of cases have been reported with co-existence of DLBCL and AITL. ${ }^{5-7}$ However, this synchronous discordant lymphoma containing AITL and EBV-positive DLBCL is extremely rare, and the prognosis is undoubtedly unfavorable.

Lenalidomide is an immunomodulatory agent with excellent therapeutic activity and tolerance in patients with relapsed/refractory (R/R) aggressive NHL. ${ }^{8}$ Lenalidomide has demonstrated significant activity alone and in combination treatment. ${ }^{8}$ Here we report a patient diagnosed with an EBV-associated discordant lymphoma who was treated successfully with a lenalidomidecombination regimen.

\section{Case Report}

A 77-year-old man was admitted to the Affiliated Hospital of Nantong University in November 2018 with a >1-month history of repeated fever, with night sweats and weight loss. Laboratory results showed thrombocytopenia and liver function reports showed increased lactate dehydrogenase and decreased albumin levels. A hepatitis B surface antigen test was positive, serum IgG levels were low, peripheral blood EBV-DNA was 2885 viral copies/mL (reference range, 0-1000 viral copies/mL), VCA IgG antibodies showed $170 \mathrm{U} / \mathrm{mL}$ (reference range, 0-20 U/mL) and EBNA IgG antibodies were 119U/mL (reference range, 0-5 U/mL). Lymphocyte subset analyses showed decreased lymphocyte, total $\mathrm{T}$ lymphocyte, helper $\mathrm{T}$ cell, and cytotoxic $\mathrm{T}$ cell counts. No obvious abnormality was detected by bone marrow puncture. Positron emission tomography (PET)/computed tomography (CT) showed increased 18F-fluorodeoxyglucose (FDG) uptake in multiple masses, including extensive lymphadenopathy in the neck, chest, abdomen, pelvic cavity, and inguinal region, with evident splenomegaly and nodular lesions, some of which showed slightly higher FDG standardized uptake values (SUVmax; approximately 10, short diameter $<2 \mathrm{~cm}$ ). In addition, an intestinal mass (measuring $5.8 \times 4.5 \times 3.7 \mathrm{~cm}$ ) revealed a notably higher FDG SUVmax of 20.5. Core needle aspiration cytology of the left axillary nodule performed the unnormal lymph node architecture with a infiltrate of atypical lymphoid proliferation, predominantly composed of medium-sized $\mathrm{T}$ cells with a clear cytoplasm, occasional eosinophils, immunoblasts, and proliferation of high endothelial venules. Immunohistochemistry showed these neoplastic $\mathrm{T}$ cells were positive for CD3, PD-1, CD10, Bcl-6, CD4, CD8,
CD5, CD21 (expansion of the follicular dendritic cell meshwork +), Ki67 $(30 \%+)$, and negativity for CD56, CD20, CXCL13, and Epstein-Barr-encoded RNA (EBER), with partial loss of CD7. T cell receptor gene rearrangement was positive but no clonal $\mathrm{IgH}$ gene rearrangement was detected (Figure 1). The patient was diagnosed with AITL, with a prognostic index for T-cell lymphoma (PIT) score of 3 . Before treatment, he suddenly developed severe abdominal pain, and abdominal CT showed gastrointestinal perforation. He therefore underwent emergency surgery, and pathological examination showed an ulcerative mass on the ileum. Immunohistochemical staining revealed that the diffuse infiltration of these abnormal medium-to-large-sized lymphocytes with prominent nucleoli was predominantly positive for Bcl-6, MUM1, CD20, CD21, CD10, EBER (>200/ high-power field), and $\mathrm{Ki} 67(80 \%+)$, but negative for CD3, PD-1, CD8, CD4, CD5, CD7, and CXCL13 (Figure 2). He was subsequently diagnosed with EBVpositive DLBCL (stage IV, group B). The International Prognostic Index (IPI) was 4, indicating high risk. The patient had a history of tuberculosis for 40 years and had undergone a left upper lobe resection 7 months prior with postoperative pathology of the lung tissue suggesting carcinoma in situ. Considering his complex illness and inferior performance status, the patient was administered six cycles of R2-miniCHOP (lenalidomide $25 \mathrm{mg}$ by oral administration on days $1-10$, rituximab $600 \mathrm{mg}$ on day 0 , cyclophosphamide $600 \mathrm{mg}$ on day 1 , doxorubicin liposome $20 \mathrm{mg}$ on day 1 , vinorelbine $30 \mathrm{mg}$ on day 1 , and prednisone $30 \mathrm{mg}$ on days 1-5), with no serious adverse events during treatment. His symptoms subsequently improved, with weight gain. PET/CT scan demonstrated decreased FDG uptake in many parts of the body compared with before treatment (Figure 3). The patient was then treated with lenalidomide $(10 \mathrm{mg} /$ day on days $1-21$, every 28 days) as maintenance therapy and remained in sustained remission for more than 20 months.

\section{Discussion}

Discordant lymphomas, which are defined as two distinct types of lymphomas coexisting at different anatomic sites, ${ }^{9}$ are relatively infrequent, particularly those containing $\mathrm{T}$ cell and $\mathrm{B}$ cell lymphomas. Here, we report a rare case of synchronous discordant lymphoma in a 77-year-old man that involved AITL and EBV-positive DLBCL that was successfully treated with a lenalidomide-combination regimen. 

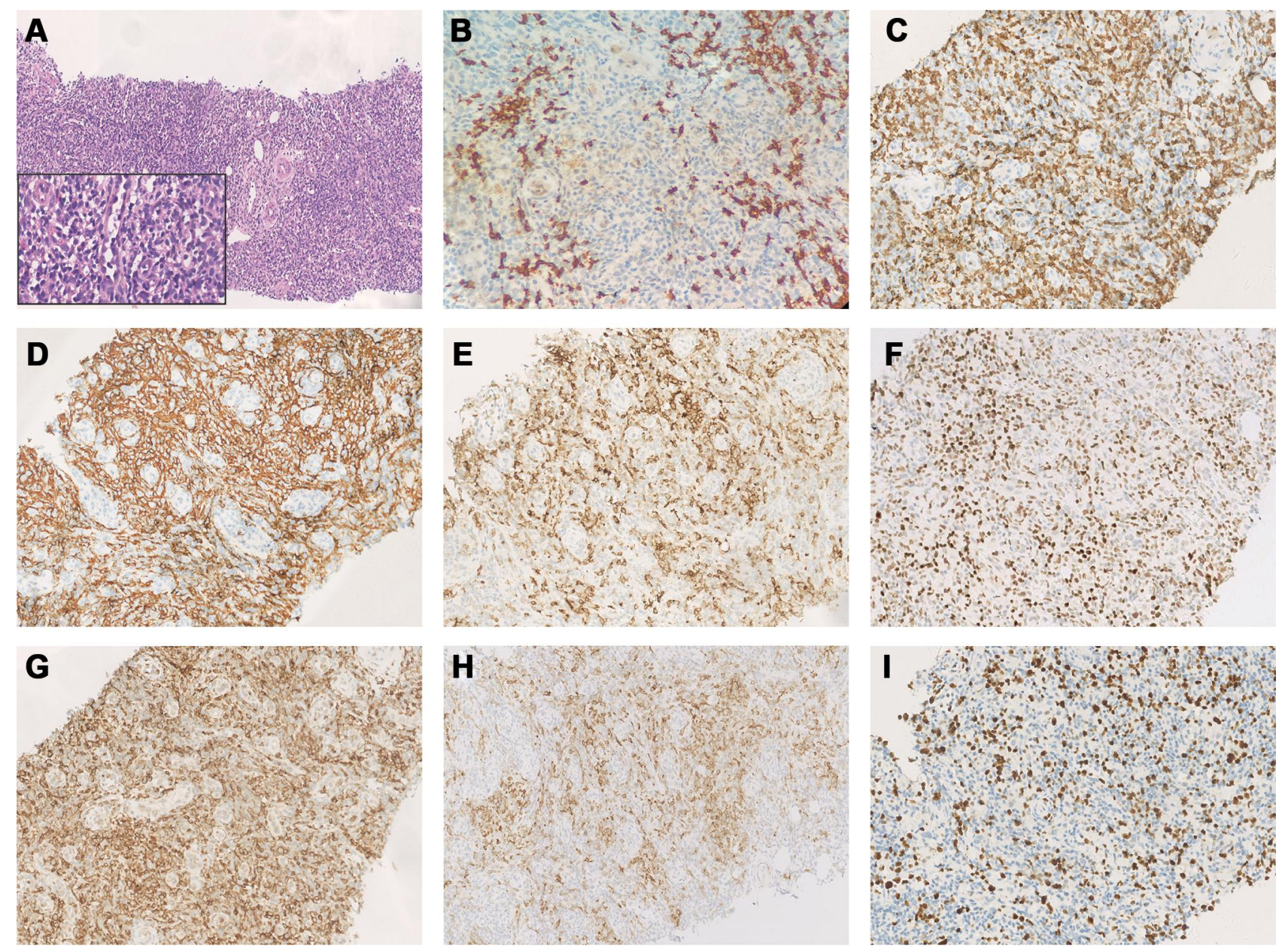

Figure I Immunohistochemical staining of angioimmunoblastic T-cell lymphoma tissue sample. Immunohistochemical staining of H\&E (A) and Ki67 (I), negative for CD20 (B) and positive for CD3 (C), CD2I (D), CDI0 (E), Bcl-6 (F), CD4 (G), PD-I (H) (A: H\&Ex 100, inset: H\&E×400, B-I: ×200).

AITL has a complex histopathological background and is thought to be derived from follicular $\mathrm{T}$ helper (TFH) cells. ${ }^{1,10}$ During normal immune function, TFH cells act as an important checkpoint for B-cell activation and differentiation, and play a role in germinal centers. ${ }^{11}$ Cytokines and chemokines released by TFH cells may direct immune cells, such as non-neoplastic responsive $\mathrm{T}$ cells, B cells, and follicular dendritic cells, into malignant tissues to form an intricate tumor microenvironment. ${ }^{12}$ TFH markers include PD1, CXCL13, inducible costimulator, Bcl-6, and $\mathrm{CD} 10 ;{ }^{1}$ however, because of its relative heterogeneity, AITL cells do not express all the characteristic markers. ${ }^{13}$ In the current case, AITL-related immunohistochemistry was negative for CXCL13. Normal TFH cells inhibit $\mathrm{T}$ cell responses via their ability to inhibit the proliferation and normal function of conventional CD4+ T cells. ${ }^{10}$ Dysregulation of TFH cells may lead to the disruption of germinal centers, associated with the occurrence of AITL. ${ }^{11}$ Patients with AITL are often immunodeficient, and their deficient $\mathrm{T}$ cell response is related to quantitative and qualitative changes in $\mathrm{T}$ cell subsets. ${ }^{10}$ A low absolute CD4+ $\mathrm{T}$ cell count was found in some cases of AITL. ${ }^{14}$ In the current case, the absolute CD4+ $\mathrm{T}$ cell count was decreased, as shown by lymphocyte subset analysis, but the above lymphocyte subsets increased after lenalidomide-combination treatment. The empty $\mathrm{T}$ cell repertoire niche left by the decrease in CD4 $+\mathrm{T}$ cells could be filled by clonal proliferation of nontumor naive $\mathrm{T}$ cells, followed by reduced complexity of the T-cell repertoire and $\mathrm{T}$ cell-mediated immunosuppression. ${ }^{14}$ Moreover, factors such as the age of the patient might also exacerbate the state of immunodeficiency. These factors could allow EBV infection or reactivation, with EBV considered to have the capability to prompt resting B cells to transform into permanent, latent lymphoblastoid cell lines. ${ }^{3,15}$ This could result in 

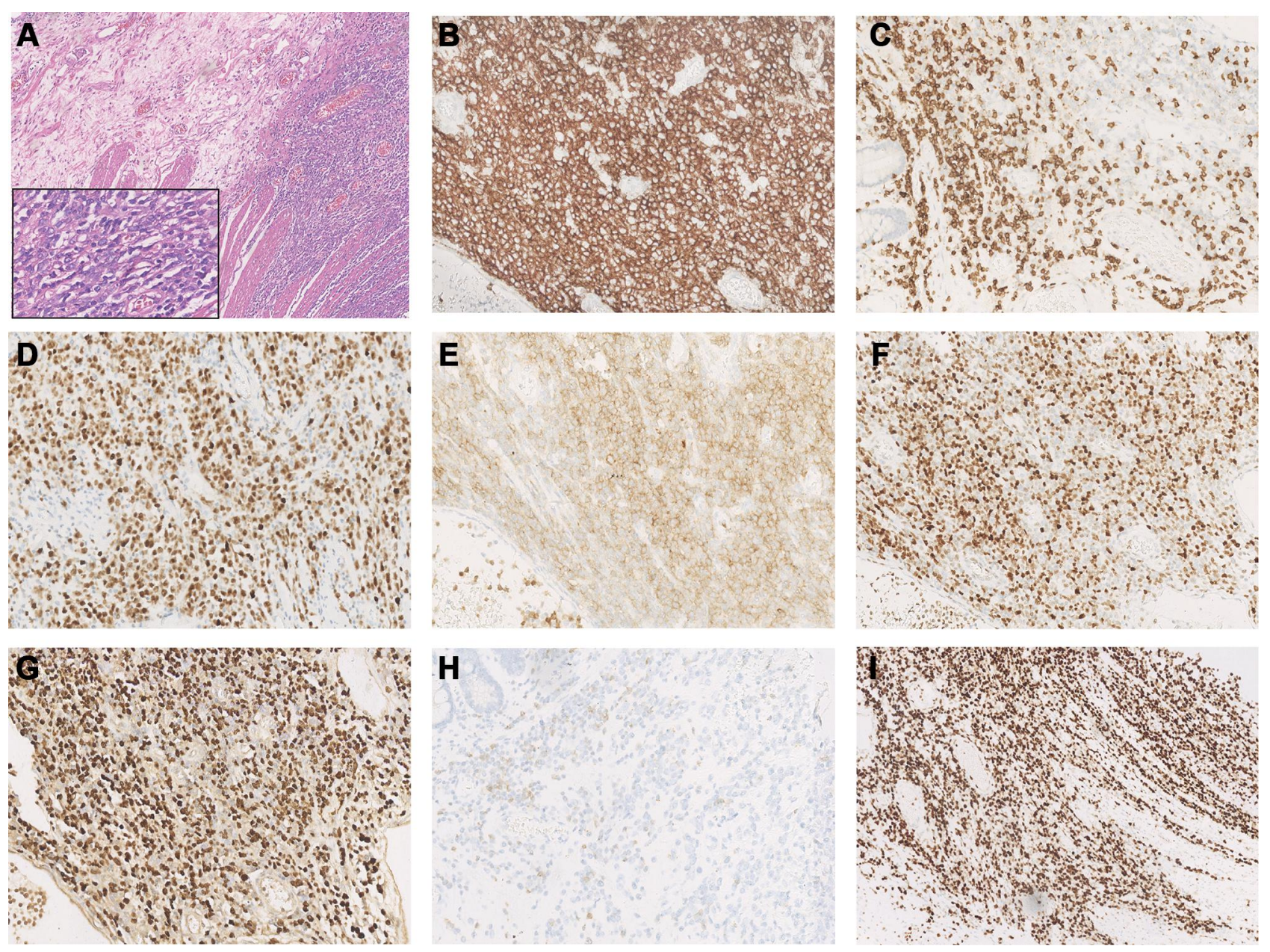

Figure 2 Immunohistochemical staining of diffuse large B-cell lymphoma tissue sample. Immunohistochemical staining of H\&E (A) and Ki67 (I), positive for CD20 (B), MUMI (D), CDIO (E), Bcl-6 (F), EBER (G) and negative for CD3 (C), PD-I (H) (A: H\&E×I00, inset: H\&E×400, B-H: $\times 200, \mathbf{I}: \times 100)$.

clonal expansion of EBV-infected B cells, followed by malignant transformation, resulting in EBV-associated B-cell lymphoma. ${ }^{16}$ The lack of clonal IgH gene rearrangement at the site of the AITL in the present patient meant that a correlation between intestinal DLBCL and AITL could not be inferred. Because serum EBV DNA and EBER were positive, EBV infection may have played a role in the formation of the discordant lymphoma in the current patient. In addition, for patients with immune deficiency, especially in the cases of iatrogenic immunosuppression or age-related immunosenescence, we should be aware of the possibility of EBV+-mucocutaneous ulcers in gastrointestinal lymphoproliferative disorders, which usually show an indolent course, manifest as ulcerative rather than mass lesions, and frequently show negative EBV in serum. ${ }^{17}$

Lenalidomide is an oral immunomodulatory drug with an acceptable toxicity profile and manageable side effects. The antitumor properties of lenalidomide in NHL include direct tumor cytotoxicity, immunoregulation, and antiangiogenesis. ${ }^{18-20}$ Previous studies have shown that lenalidomide can arrest tumor cells in the G0/G1 phase of the cell cycle by up-regulating $\mathrm{p} 21^{\mathrm{WAF}-1}$, related to its antiproliferative effect. ${ }^{18,21}$ In the case of malignant lymphoma B cells, the antineoplastic effect of lenalidomide might be associated with down-regulation of interferon regulatory factor 4 and nuclear factor- $\kappa \mathrm{B}$ pathway inhibition. Research findings also suggest that lenalidomide can repair T-cell immune synapse formation, stimulate T-cell proliferation, and enhance the cytotoxicity of $\gamma \delta$ T cells. ${ }^{21}$ In addition to the above mechanisms of action, lenalidomide also functions in the microenvironment to increase the number of natural killer (NK) cells and enhance their cytotoxic effect, increase antibody-dependent cellular cytotoxicity (ADCC), and regulate cytokines that may be related to the inflammatory reaction, tumor cell proliferation, and metastasis. ${ }^{21}$ Lenalidomide may also act synergistically with other drug therapies, such as rituximab. By 


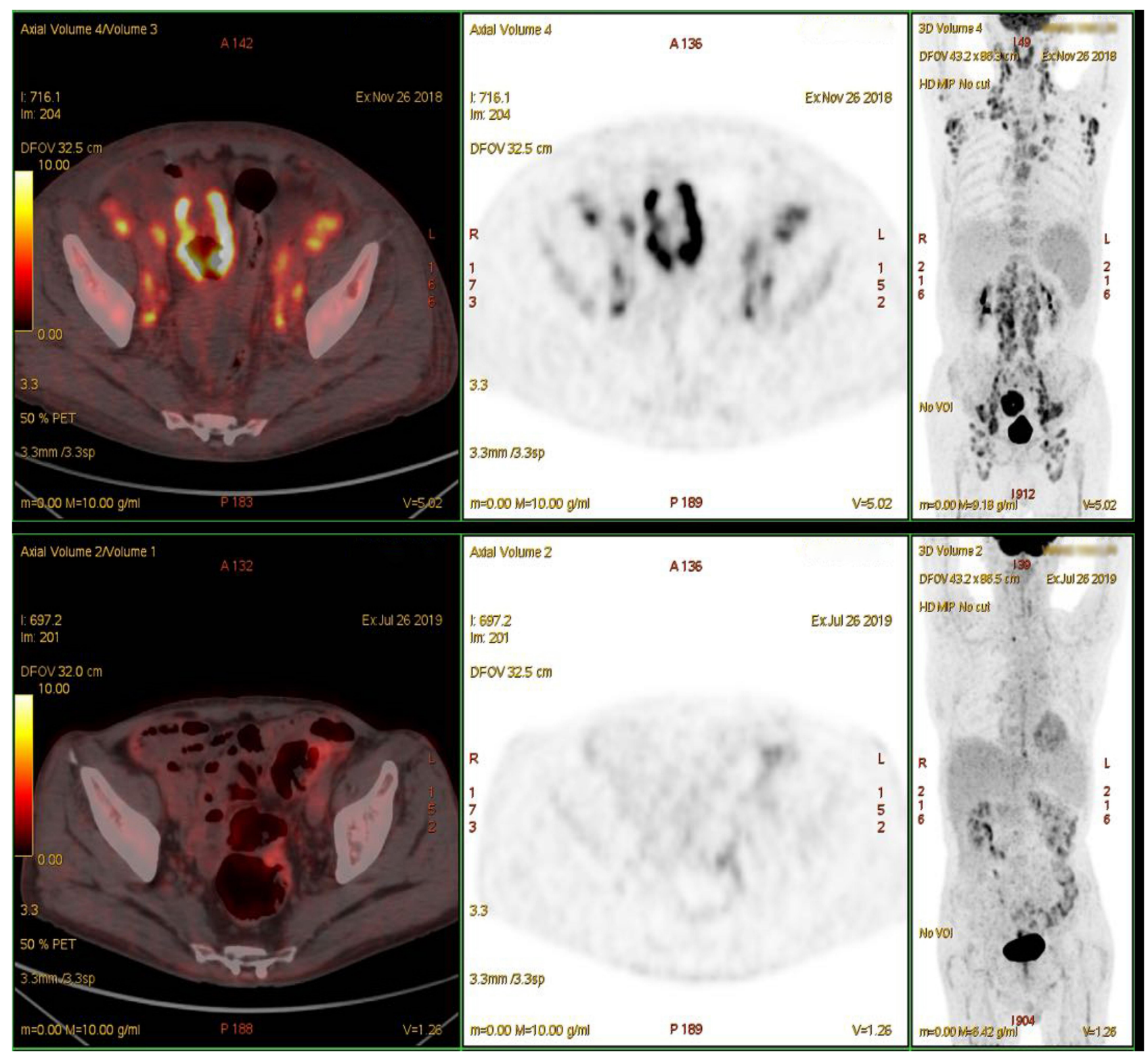

Figure 3 Comparison of positron emission tomography/computed tomography scans before and after lenalidomide-combination treatment.

enhancing CD16 expression, lenalidomide can strengthen the rituximab-dependent NK cell-mediated ADCC. ${ }^{18,21}$ It can also up-regulate the phosphorylation of c-Jun $\mathrm{N}$-terminal protein kinases and activate mitochondrial pathways of apoptosis to enhance rituximab-induced apoptosis. ${ }^{18,21}$ Several studies showed that lenalidomide had strong therapeutic activity in patients with $\mathrm{R} / \mathrm{R}$ DLBCL, and its early application improved disease control and significantly reduced the occurrence of refractory disease in patients with B-NHL. ${ }^{18,22}$ The NHL-003 study of 217 patients with aggressive R/R NHL (108 DLBCL) treated with lenalidomide monotherapy showed an overall response rate (ORR) of $28 \%$ in DLBCL patients. ${ }^{8}$
Furthermore, the REAL07 study showed that $86 \%$ of 49 elderly patients with untreated DLBCL achieved complete remission after six cycles of R2-CHOP, with no grade 4 non-hematological adverse events. In addition to B cell lymphoma, lenalidomide has also demonstrated therapeutic activity in T cell lymphoma. ${ }^{8}$ The EXPECT trial treated 54 patients with R/R peripheral T cell lymphomas (including 26 AITL) with lenalidomide monotherapy, and found an ORR in the AITL subgroup of $31 \%{ }^{8}$ Lenalidomide was also shown the possible to reactivate the EBV lytic cycle, causing tumor cells to attract more humoral and cytotoxic $\mathrm{T}$ cell responses, resulting in cell killing. ${ }^{23}$ Lenalidomide may therefore also have therapeutic effects in patients with 
EBV-associated lymphoma. Considering the mechanism of action of lenalidomide and its demonstrated clinical efficacy, we decided to use lenalidomide-combination therapy in the current patient, with exciting results.

$\mathrm{PET} / \mathrm{CT}$ is a non-invasive examination method with high sensitivity for whole-body oncologic imaging, can be used to select areas for biopsy and potentially identify high-risk patients before starting therapy. The SUV is an independent biomarker in lymphomas. Previous studies among patients with mixed NHL subtypes recommended that high SUVmax values $(>10)$ may predict aggressive NHL, while FDG uptake intensities differed among lymphoma subtypes. A study of 111 patients diagnosed with lymphoma concluded that the estimated median SUVmax was higher in patients with aggressive B-NHL compared with those with indolent B-NHL (17.0 vs 8.3), while the estimated median SUVmax values of T-NHL and Hodgkin lymphoma (9.9 and 12.4, respectively) were intermediate between these two groups. $^{24-26}$ A high SUV is generally thought to be related to a heavy tumor burden and high biological invasiveness, and most EBV-related lymphoid neoplasms show high FDG-avidity in PET/CT. In the current case, PET/CT showed significantly different SUVs, particularly in the intestinal EBV-positive DLBCL (SUVmax 20.5, Ki67 $70 \%$ ). We considered that the high FDG uptake here might have been associated with EBV infection. A study of 205 patients with confirmed gastric cancer (including $7.3 \%$ with EBV-positive gastric cancer) found a significant difference in mean SUVmax values between the EBV-positive and negative groups. ${ }^{27}$ Recently, Zhang et al found that EBV latent membrane protein 1 (LMP1) upregulated transcription of the glucose transporter 1 to control aerobic glycolysis and tumor growth in nasopharyngeal carcinoma. ${ }^{27}$ In addition, locally metabolically active inflammatory cells may also increase FDG uptake. ${ }^{27}$ Interestingly, Sun et al recently reported their case diagnosed with a discordant lymphoma whose PET-CT showed increased FDG uptake widely with a noteworthy SUVmax of 25.6 at the stomach, the pathological biopsy of the gastric mucosa showed DLBCL, different from AITL showed in inguinal lymph node. ${ }^{28}$ Since lymphomas can occur in different parts of the body, multi-site puncture biopsy may be required, especially in patients for whom imaging shows wide variation in SUVmax values among different sites. Clinically, repeated lymph node biopsies are rarely performed in patients with a definitive diagnosis of lymphoma, which may underestimate the condition of discordant cases.

\section{Conclusion}

Herein, we demonstrated the successful use of lenalidomide-combination therapy in a patient with a discordant lymphoma consisting of AITL and EBV-positive DLBCL. This case highlights the importance of histopathological analysis of multi-site puncture biopsies, especially in patients in whom PET/CT shows wide differences in SUVmax values at different sites. Comprehensive evaluation is needed to avoid a missed diagnosis and to ensure an appropriate treatment regimen. Moreover, this case demonstrates the potential use for lenalidomide in patients with an EBV-associated discordant lymphoma. However, further studies with longer follow-up and clinical trials are needed to evaluate the efficacy and adverse reactions of lenalidomide therapy.

\section{Ethics Approval and Consent to Participate}

This study was approved by the Ethical Committee of the Affiliated Hospital of Nantong University.

\section{Consent for Publication}

Written informed consent was obtained from the patient for the publication of this case report and any accompanying images. A copy of the consent form is available for review by the Editor of this journal.

\section{Acknowledgments}

We thank Susan Furness, PhD and Gabrielle White Wolf, $\mathrm{PhD}$, from Liwen Bianji, Edanz Editing China (www. liwenbianji.cn/ac), for editing the English text of a draft of this manuscript. Pan $\mathrm{Hu}$ and $\mathrm{Yu}$ Ben are co-first authors.

\section{Funding}

This study was funded by the National Natural Science Foundation international cooperation (81570184), the Science and Technology Project of Nantong City (MS22018008), the Science and Technology Project of Nantong City (MS12017003-2), China Postdoctoral Science Foundation (2019M660127), Jiangsu Province Postdoctoral Science Foundation (2019K062), Jiangsu Province Postdoctoral Foundation (2019Z146).

\section{Disclosure}

The authors report no conflicts of interest in this work. 


\section{References}

1. Szablewski V, Dereure O, Rene C, et al. Cutaneous localization of angioimmunoblastic T-cell lymphoma may masquerade as B-cell lymphoma or classical Hodgkin lymphoma: a histologic diagnostic pitfall. J Cutan Pathol. 2019;46(2):102-110. doi:10.1111/cup.13382

2. Ellis C, Ramirez J, LaFond AA. Angioimmunoblastic T-cell lymphoma mimicking diffuse large B-cell lymphoma. Cutis. 2018;102 (3):179-182.

3. Takahashi T, Maruyama R, Mishima S, et al. Small bowel perforation caused by Epstein-Barr virus-associated B cell lymphoma in a patient with angioimmunoblastic T-cell lymphoma. J Clin Exp Hematop. 2010;50(1):59-63. doi:10.3960/jslrt.50.59

4. Zhou Y, Xu Z, Lin W, et al. Comprehensive genomic profiling of EBV-positive diffuse large B-cell lymphoma and the expression and clinicopathological correlations of some related genes. Front Oncol. 2019;9:683. doi:10.3389/fonc.2019.00683

5. Poon F, Ieremia E, Collins G, Matin RN. Epstein-Barr virus-induced cutaneous diffuse large B-cell lymphoma in a patient with angioimmunoblastic T-cell lymphoma. Am J Dermatopathol. 2019;41 (12):927-930. doi:10.1097/DAD.0000000000001371

6. Hong R, Sheng L, Ouyang G. Composite angioimmunoblastic T cell/ diffuse large B-cell lymphoma treated with reduced-intensity conditioning HLA-haploidentical allo-HSCT: a case report and review of the literature. Int J Clin Exp Pathol. 2018;11(11):5473-5480.

7. Zhou Y, Rosenblum MK, Dogan A, Jungbluth AA, Chiu A. Cerebellar EBV-associated diffuse large B cell lymphoma following angioimmunoblastic $\mathrm{T}$ cell lymphoma. J Hematop. 2015;8 (4):235-241. doi:10.1007/s12308-015-0241-8

8. Yamshon S, Ruan J. IMiDs New and Old. Curr Hematol Malig Rep. 2019;14(5):414-425. doi:10.1007/s11899-019-00536-6

9. Zhang C, Yi Y, Chen C, Wang J, Liu Z. Discordant lymphoma consisting of mediastinal large B-cell lymphoma and nodular sclerosis Hodgkin lymphoma in the right supraclavicular lymph nodes: a case report. Diagn Pathol. 2015;10(1):215. doi:10.1186/s13000015-0450-6

10. de Leval L, Gisselbrecht C, Gaulard P. Advances in the understanding and management of angioimmunoblastic T-cell lymphoma. $B r J$ Haematol. 2010;148(5):673-689. doi:10.1111/ j.1365-2141.2009.08003.x

11. Lunning MA, Vose JM. Angioimmunoblastic T-cell lymphoma: the many-faced lymphoma. Blood. 2017;129(9):1095-1102. doi:10.1182/ blood-2016-09-692541

12. Mhaidly R, Krug A, Gaulard P, Lemonnier F, Ricci JE, Verhoeyen E. New preclinical models for angioimmunoblastic T-cell lymphoma: filling the GAP. Oncogenesis. 2020;9(8):73. doi:10.1038/s41389-02000259-x

13. Eivazi S, Bagheri S, Hashemzadeh MS, et al. Development of $\mathrm{T}$ follicular helper cells and their role in disease and immune system. Biomed Pharmacother. 2016;84:1668-1678.

14. Huang J, Zhang PH, Gao YH, Qiu LG. Sequential development of diffuse large B-cell lymphoma in a patient with angioimmunoblastic T-cell lymphoma. Diagn Cytopathol. 2012;40(4):346-351. doi: $10.1002 / \mathrm{dc} .21641$

OncoTargets and Therapy

\section{Publish your work in this journal}

OncoTargets and Therapy is an international, peer-reviewed, open access journal focusing on the pathological basis of all cancers, potential targets for therapy and treatment protocols employed to improve the management of cancer patients. The journal also focuses on the impact of management programs and new therapeutic

Submit your manuscript here: https://www.dovepress.com/oncotargets-and-therapy-journa
15. Mosalpuria K, Bociek RG, Vose JM. Angioimmunoblastic T-cell lymphoma management. Semin Hematol. 2014;51(1):52-58. doi:10.1053/j.seminhematol.2013.11.008

16. Wang Y, Xie B, Chen Y, Huang Z, Tan H. Development of angioimmunoblastic T-cell lymphoma after treatment of diffuse large B-cell lymphoma: a case report and review of literature. Int J Clin Exp Pathol. 2014;7(6):3432-3438.

17. Alruwaii ZI, Montgomery EA. Select Epstein-Barr virus-associated digestive tract lesions for the practicing pathologist. Arch Pathol Lab Med. 2020. doi:10.5858/arpa.2019-0703-RA

18. Garciaz S, Coso D, Schiano de Colella JM, Bouabdallah R. Lenalidomide for the treatment of B-cell lymphoma. Expert Opin Investig Drugs. 2016;25(9):1103-1116. doi:10.1080/ 13543784.2016.1208170

19. Chen N, Zhou S, Palmisano M. Clinical pharmacokinetics and pharmacodynamics of lenalidomide. Clin Pharmacokinet. 2017;56 (2):139-152. doi:10.1007/s40262-016-0432-1

20. Witzig TE, Nowakowski GS, Habermann TM, et al. A comprehensive review of lenalidomide therapy for B-cell non-Hodgkin lymphoma. Ann Oncol. 2015;26(8):1667-1677. doi:10.1093/annonc/mdv102

21. Gribben JG, Fowler N, Morschhauser F. Mechanisms of action of lenalidomide in B-cell non-Hodgkin lymphoma. J Clin Oncol. 2015;33(25):2803-2811. doi:10.1200/JCO.2014.59.5363

22. Miao Y, Medeiros LJ, Li Y, Li J, Young KH. Genetic alterations and their clinical implications in DLBCL. Nat Rev Clin Oncol. 2019;16 (10):634-652.

23. Jones RJ, Iempridee $\mathrm{T}$, Wang $\mathrm{X}$, et al. Lenalidomide, thalidomide, and pomalidomide reactivate the Epstein-Barr virus lytic cycle through phosphoinositide 3-kinase signaling and Ikaros expression. Clin Cancer Res. 2016;22(19):4901-4912. doi:10.1158/1078-0432. CCR-15-2242

24. Ngeow JYY, Quek RHH, Ng DCE, et al. High SUV uptake on FDG-PET/CT predicts for an aggressive B-cell lymphoma in a prospective study of primary FDG-PET/CT staging in lymphoma. Ann Oncol. 2009;20(9):1543-1547. doi:10.1093/annonc/mdp030

25. Giraudo C, Karanikas G, Weber M, et al. Correlation between glycolytic activity on [18F]-FDG-PET and cell density on diffusion-weighted MRI in lymphoma at staging. J Magn Reson Imaging. 2018;47(5):1217-1226. doi:10.1002/jmri.25884

26. Mir F, Barrington SF, Brown H, et al. Baseline SUVmax did not predict histological transformation in follicular lymphoma in the Phase 3 GALLIUM study. Blood. 2020;135(15):1214-1218. doi:10.1182/blood.2019001091

27. Na SJ, Park HL, Lee SY, Song KY, Kim SH. Correlation between infection status of Epstein-Barr virus and (18)F-fluorodeoxyglucose uptake in patients with advanced gastric cancer. In Vivo. 2017;31 (4):749-753.

28. Sun JN, Tan YH, Li YY, et al. [Discordant lymphoma consisting of angioimmunoblastic T-cell lymphoma and diffuse large B-cell lymphoma: a case report]. Zhonghua Xиe Ye Xue Za Zhi. 2020;41(9):777. Chinese. agents and protocols on patient perspectives such as quality of life, adherence and satisfaction. The manuscript management system is completely online and includes a very quick and fair peer-review system, which is all easy to use. Visit http://www.dovepress.com/ testimonials.php to read real quotes from published authors. 